\title{
High-level diterpene production by transient expression in Nicotiana benthamiana
}

\author{
Kathleen Brückner and Alain Tissier ${ }^{*}$
}

\begin{abstract}
Background: Characterization of plant terpene synthases is typically done by production of recombinant enzymes in Escherichia coli. This is often difficult due to solubility and codon usage issues. Furthermore, plant terpene synthases which are targeted to the plastids, such as diterpene synthases, have to be shortened in a more or less empirical approach to improve expression. We report here an optimized Agrobacterium-mediated transient expression assay in Nicotiana benthamiana for plant diterpene synthase expression and product analysis.

Results: Agrobacterium-mediated transient expression of plant diterpene synthases in $\mathrm{N}$. benthamiana led to the accumulation of diterpenes within 3 days of infiltration and with a maximum at 5 days. Over $50 \%$ of the products were exported onto the leaf surface, thus considerably facilitating the analysis by reducing the complexity of the extracts. The robustness of the method was tested by expressing three different plant enzymes, cembratrien-ol synthase from Nicotiana sylvestris, casbene synthase from Ricinus communis and levopimaradiene synthase from Gingko biloba. Furthermore, co-expression of a 1-deoxy-D-xylulose-5-phosphate synthase from tomato and a geranylgeranyl diphosphate synthase from tobacco led to a 3.5-fold increase in the amount of cembratrien-ol produced, with maximum yields reaching $2500 \mathrm{ng} / \mathrm{cm}^{2}$.

Conclusion: With this optimized method for diterpene synthase expression and product analysis, a single infiltrated leaf of $N$. benthamiana would be sufficient to produce quantities required for the structure elucidation of unknown diterpenes. The method will also be of general use for gene function discovery, pathway reconstitution and metabolic engineering of diterpenoid biosynthesis in plants.
\end{abstract}

Keywords: Diterpene synthase, Agrobacterium, Transient protein expression, Nicotiana benthamiana

\section{Background}

Terpenoids constitute a highly diverse group of compounds which are found in a wide variety of plants and microbial species as well as in animals. Their structural complexity is based on two comparatively simple basic C5 building blocks, isopentenyl diphosphate (IPP) and dimethylallyl diphosphate (DMAPP). Sequential headto-tail coupling of IPP to allylic isoprenyl diphosphates, starting with DMAPP, gives rise to isoprenyl moieties of increasing lengths, namely geranyl diphosphate (GPP, $\mathrm{C}_{10}$ ), farnesyl diphosphate (FPP, $\mathrm{C}_{15}$ ) and geranylgeranyl diphosphate (GGPP, $\mathrm{C}_{20}$ ). These isoprenyl diphosphates are the substrates of a large class of enzymes, the terpene synthases (TPS), which, through their cyclase activity, are the key contributors to the structural diversity of

\footnotetext{
* Correspondence: Alain.Tissier@ipb-halle.de

Department of Cell and Metabolic Biology, Leibniz Institute of Plant Biochemistry, Weinberg 3, 06120 Halle-Saale, Germany
}

terpenes. Accordingly, terpenes are classified according to the number of isoprenyl units they contain in monoterpenes $\left(C_{10}\right)$, sesquiterpenes $\left(C_{15}\right)$ and diterpenes $\left(C_{20}\right)$. Many terpenoid compounds are of great importance for human nutrition, fragrance industry and medicine. Several plant diterpenoids in particular are produced commercially, such as the anti-cancer compound Taxol, the labdanoid sclareol as a precursor for fragrance ingredient, or carnosic acid and carnosol as anti-oxidants for cosmetic and food applications [1-3]. To increase the yield of these diterpenoids or to gain better access to the diversity of diterpenoids by metabolic engineering in microbial or plant hosts there is an increasing interest in elucidating biosynthesis pathway genes.

Escherichia coli has been typically employed for the biochemical characterization of terpene synthases because of the ease of manipulation and of the access to a wide range of expression hosts and vectors. A large 
number of plant mono- and sesquiterpene synthases have been successfully expressed in E. coli and isolated for in vitro enzyme assays [4]. Heterologous protein expression using microbial hosts has also been widely applied for the screening of diterpene synthase function. One of the best studied example is taxadiene synthase from Taxus sp. which synthesizes taxa-4(5),11(12)-diene, a key intermediate for the biosynthesis of the anticancer drug paclitaxel (Taxol) $[5,6]$. By using recombinant E. coli strains, some further diterpene synthases have been identified, such as $z$-abienol synthase from Nicotiana tabacum and bifunctional levopimaradiene/abietadiene synthases from Pinus $s p$., respectively $[7,8]$.

However, using microbial hosts presents some drawbacks for overexpression of plant terpene synthases. In particular, the proteins may not fold properly and form aggregates that are stored in inclusion bodies as reported for casbene cyclase production in E. coli [9]. Misfolding could be caused by the presence of N-terminal signal peptides which are non-functional in microbial hosts. Foreign protein production in yeast and E. coli can also be accompanied by difficulties in plasmid stability and codon usage. Furthermore, toxicity of the target gene sequence or of the catalytic activity of the protein in combination with already low expression levels could prevent the production of functional recombinant protein. Nonetheless, there have been successful attempts at producing diterpenes in vivo in E. coli or yeast hosts $[10,11]$. In these cases however, the production of terpenoids can be limited by intracellular levels of GGPP or toxicity of the diterpenoids to the host. Most prokaryotes including E. coli and plant plastids synthesize IPP and DMAPP through the 2-C-methyl-D-erythritol4-phosphate (MEP) pathway while most eukaryotes synthesize IPP via the mevalonate (MEV) pathway. Increasing the flux by single-enzyme introduction or exogenous precursor supply may improve productivity [12]. On the other hand, the introduction of a MEV pathway in $E$. coli was reported to induce growth inhibition triggered by the accumulation of HMG-CoA [13], but successive improvements led to high production levels of amorphadiene, the precursor of the anti-malaria compound, artemisinin $[14,15]$. Yeast is an attractive alternative to E. coli, in particular because the expression of ER-bound plant cytochrome P450 oxygenases, which are frequently involved in the biosynthesis pathways of plant terpenoids, is more easily implemented in yeast than in E. coli due to the absence of ER in bacteria. Thus a number of reports describe the use of yeast to produce plant diterpenoids [16-22]. So far, the production yields of diterpenes in yeast rarely exceed several hundred $\mathrm{mg} / \mathrm{mL}$, thus far below the rates achieved for the production of artemisinic acid in yeast $(25 \mathrm{~g} / \mathrm{L})$ [23]. The reasons for this are not clear but could have to do with general toxicity of diterpenes towards yeast. Furthermore, since plant diterpene synthases are usually targeted to the plastids, optimization of expression in yeast is achieved through empirical shortening of the target peptide.

Nicotiana benthamiana, which is now widely used for transient expression assays in plants, is an alternative host which may circumvent the need for optimization required with microbial hosts. Since plants have both functional MEV and MEP pathways, extensive engineering of these pathways can be avoided to detect the products of terpene synthases. Furthermore, expression in planta allows investigating issues such as sub-cellular localization. $N$. benthamiana has originally been used as host in plant virology, and is now widely employed for transient protein expression [24]. The method of agroinfiltration is highly efficient in $N$. benthamiana and permits proteins of interest to be produced transiently in plant cells $[25,26]$. Recently, $N$. benthamiana was used successfully for the production of plant terpene synthases, namely amorpha-4,11-diene synthase and epi-cedrol synthase from Artemisia annua [27,28]. These investigations revealed that $N$. benthamiana can be used to produce recombinant biosynthetic enzymes in relatively high yields of 90 and $96 \mathrm{mg}$ protein per $\mathrm{kg}$ fresh weight of infiltrated leaves, respectively. Expression in $N$. benthamiana was also used successfully for examining the biochemical function of plant mono-TPS linalool synthase ApLS1 from kiwifruit [29], and of several sesquiTPS enzymes $[27,30]$ by directly detecting the products, thus bypassing the need of in vitro assays. This approach was also effective for the expression of triterpene biosynthesis enzymes including an acyl-transferase and a multifunctional cytochrome P450 [31,32].

These considerations led us to adapt the $N$. benthamiana transient expression system for the functional analysis of diterpene synthases. A simple and efficient method for in planta expression of various plant diterpene synthases including the rapid detection of the corresponding products by GC-MS is described.

\section{Results}

High-level diterpene yield in exudate of agro-infiltrated $N$. benthamiana leaves

To test $N$. benthamiana as a transient expression system for the production of plant diterpenes, we chose the cembratrien-ol (CBT-ol) synthase encoded by the trichome-specific NsCBTS2a gene from Nicotiana sylvestris [33]. CBTS enzymes from tobacco are class I terpene synthases which directly cyclize GGPP to a mixture of $\alpha$ and $\beta$-CBT-ol.

Agrobacterium strains containing T-DNA constructs with the full length CBTS2a coding sequence under the control of a Cauliflower Mosaic Virus (CaMV) $35 \mathrm{~S}$ promoter were used to infiltrate $N$. benthamiana leaves. 
CBTS2a was co-expressed with viral-encoded protein p19 that has been shown to suppress post-transcriptional gene silencing in host cells thereby allowing high levels of transient expression [26]. Five days after infiltration, the surface of leaf discs from the infiltrated parts was extracted with hexane and analyzed by GC-MS. The CBT-ol peak was clearly detectable (Figure $1 \mathrm{~A}$ ) and identified by comparison of mass spectra (see Additional file 1) with published references and extracts of $N$. tabacum cultivars which produce significant amounts of CBT-ol [8,33]. A time-course analysis confirmed that CBT-ol accumulates to significant amounts 3 days post-infiltration and strongly increases 5 days post-infiltration (Figure 1C).

To see what portion of CBT-ol produced during transient expression remains in the leaf, extracts from leaves which had been washed with hexane were prepared and the CBT-ol content was estimated by GC-MS (Figure 1B, see Additional file 2 and Additional file 3). In comparison to the CBT-ol yield in hexane washes, a slightly larger portion of CBT-ol remained inside the leaves three days post-infiltration (Figure 1C). Five days after infiltration however, comparable amounts were detected from the internal and secreted fractions. As few as six leaf discs (diameter $9 \mathrm{~mm}$ ) from infiltrated areas of $N$. benthamiana were required for the detection of CBT-ol from leaf washes.

\section{Efficient expression of various plant diterpene synthases}

To test the robustness of our assay, other diterpene synthases were expressed under the same conditions. Ricinus communis casbene cyclase (RcCS) was first tested and co-expressed with p19. Like NsCBTS2a, RcCS is a class I terpene synthase. It catalyzes the cyclization of GGPP to casbene, a diterpene phytoalexin with antifungal activity in germinating seedlings of castor bean [34]. Casbene was readily detected in hexane washes from
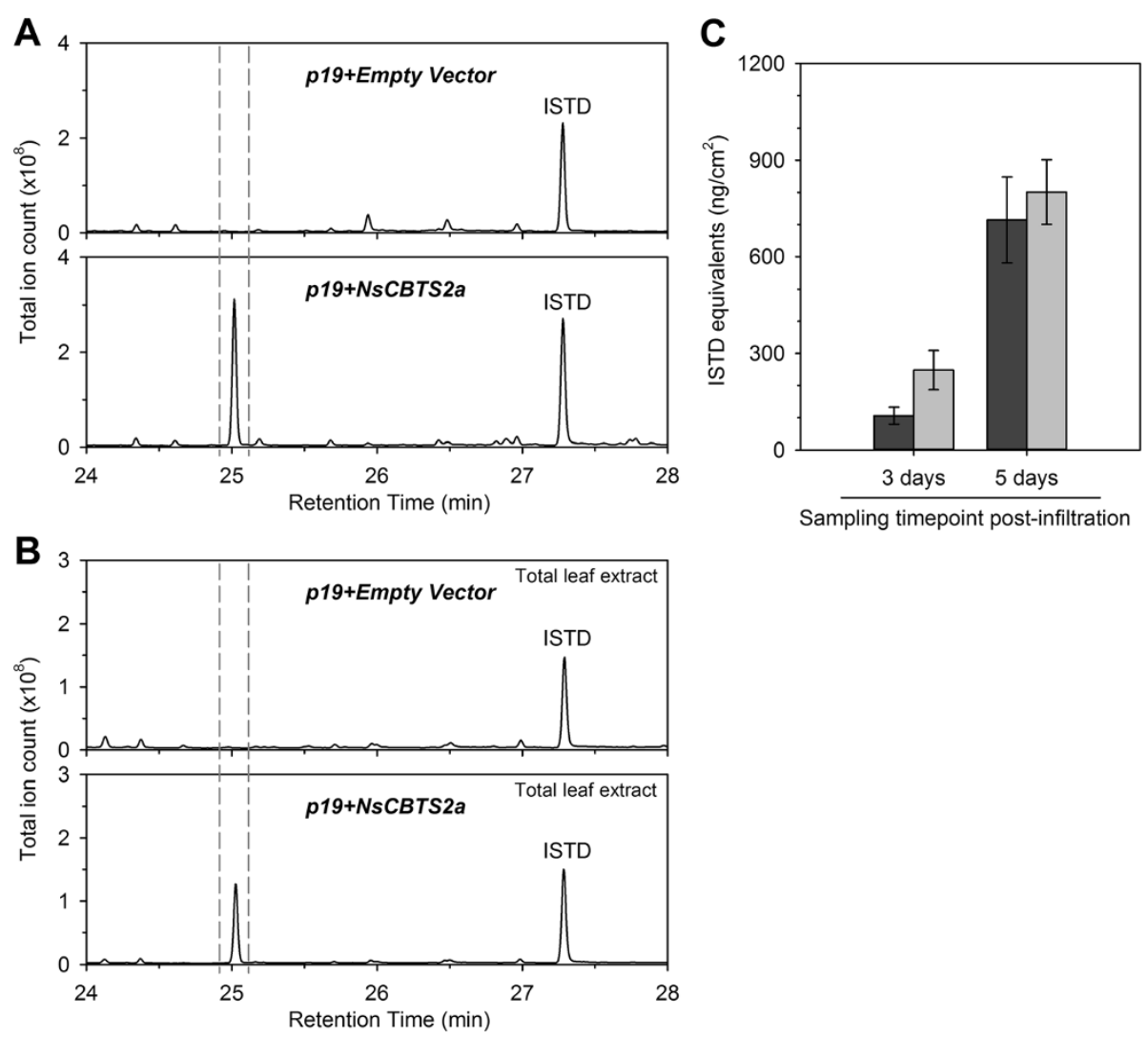

Figure 1 Production of CBT-ol. (A) Total ion chromatograms of hexane washes from agro-infiltrated N. benthamiana leaves co-expressing p19 and NSCBTS2a or the empty T-DNA vector (pL1F-1) as a control. The CBT-ol peak is framed by vertical dashed lines. The a- and $\beta$-stereoisomers of CBT-ol could not be separated under standard GC-MS conditions. (B) Total ion chromatograms of total extracts from agro-infiltrated N. benthamiana leaves. The extracts were purified over SPE column prior to GC-MS analysis. (C) CBT-ol content in agro-infiltrated leaves harvested three and five days after transformation of $N$. benthamiana plants with NsCBTS2a and p19. Hexane washes from the surface of treated leaves (black bars) and hexane extracts from the rest of the leaf material (total extracts, grey bars) were analyzed by GC-MS. No CBT-ol was detected in leaves transformed with the empty T-DNA vector (see Additional file 3). Samples were taken from two N. benthamiana plants, in each case from two infiltrated leaves. Mean CBT-ol values \pm standard error of the mean $(S E M)$ are presented $(n=8)$. There is a statistically significant difference $(P<0.001$, two-way-ANOVA) in the mean CBT-ol values regarding the timepoint of sampling post-infiltration. ISTD - internal standard sclareol. 
treated tissue five days post-infiltration, as indicated by GC-MS analysis (Figure 2A). Lacking commercially available standards, confirmation of casbene identity was based on the comparison of experimental mass spectra (see Additional file 1) with published spectra for this compound [10]. Simple leaf dipping was sufficient for the purification of up to $227 \mathrm{ng} / \mathrm{cm}^{2}$ casbene (in sclareol equivalent) from treated tissue (Figure 2B).
Levopimaradiene synthase from Ginkgo biloba (GbLS) was expressed under the same conditions. This bifunctional diterpene synthase catalyzes a multi-step reaction in which the precursor of the pharmaceutically important ginkgolides, levopimaradiene, is formed [35]. Upon expression of GbLS in $N$. benthamiana, levopimaradiene was produced and identified (Figure 2A, see Additional file 1) based on published references [35,36]. Noticeably,
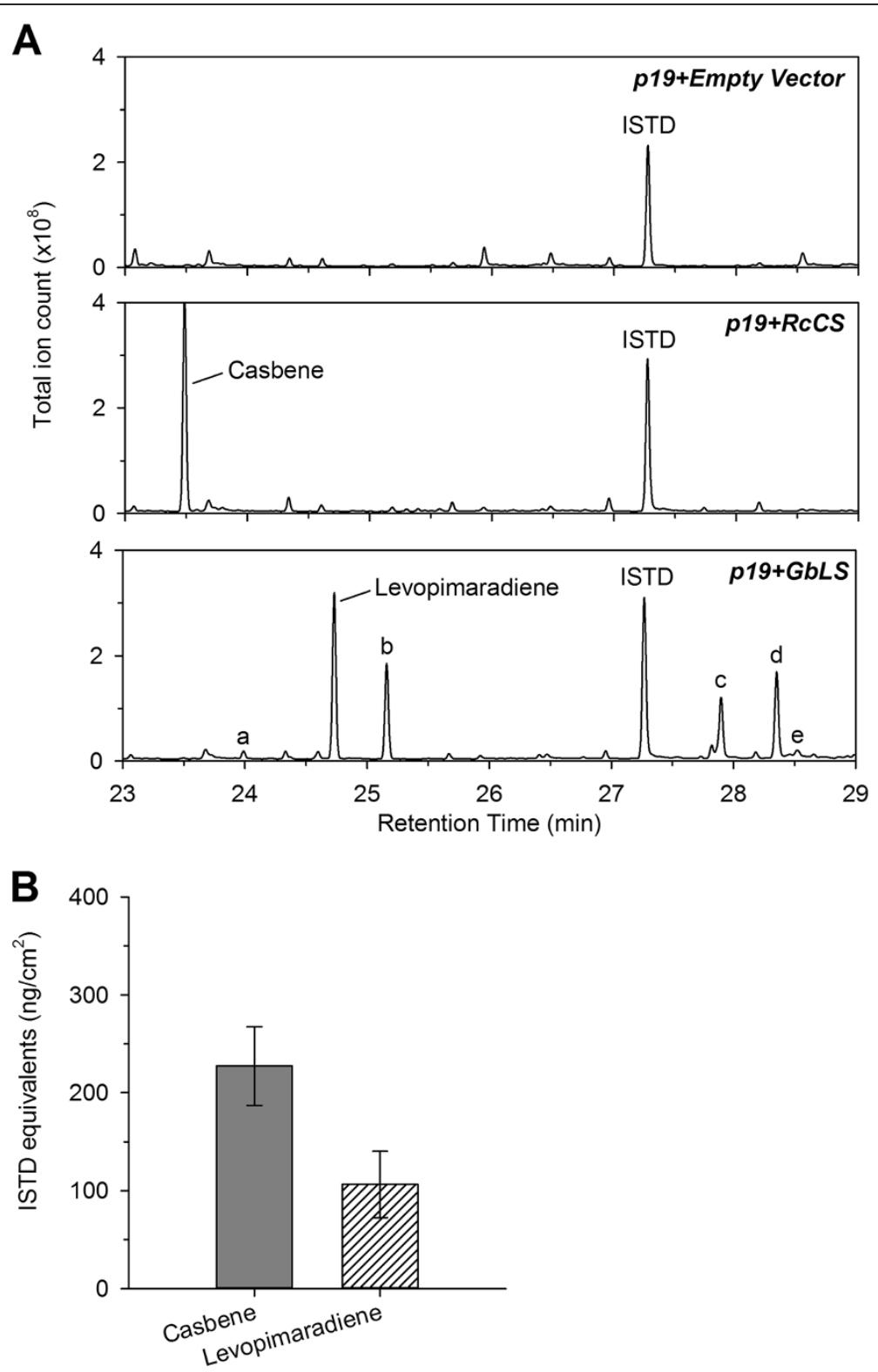

Figure 2 Production of casbene and of levopimaradiene. (A) Total ion chromatograms of hexane washes from $\mathrm{N}$. benthamiana leaves agro-infiltrated with p19 and either the empty T-DNA vector (control) or RcCS or GbLS. Peaks for casbene, levopimaradiene and other novel products $(\mathrm{a}-\mathrm{e})$ are indicated. (B) Casbene and levopimaradiene content in hexane washes from leaves agro-infiltrated with p19 and RcCS or GbLS, respectively. Samples were taken five days post-infiltration from two individual $N$. benthamiana plants, each of them having two completely infiltrated leaves. Mean casbene and levopimaradiene values \pm SEM are presented ( $n=16$, two independent experiments). Sclareol was used as internal standard (ISTD) for quantification. 
the yield of levopimaradiene in surface extracts of infiltrated leaves was lower compared to those observed for CBT-ol and casbene upon transient expression of the corresponding enzymes, and rarely exceeded $100 \mathrm{ng} / \mathrm{cm}^{2}$ five days post-infiltration (Figure 2B). The expression of LS in N. benthamiana further resulted in the formation of other diterpene products (Figure 2A, peaks a and b). One of those compounds was identified as abietatriene (peak b, see Additional file 4) which has been reported previously as a dehydrogenated derivative of levopimaradiene and found in roots of Gingko biloba seedlings [37]. The identity of peak a could not be defined. Its mass spectrum (see Additional file 4) looked highly similar to that of sandaracopimaradiene which was detected in culture media of an engineered $E$. coli strain expressing LS [38], and to that of a pimaradiene-like hydrocarbon which was found in roots of Ginkgo biloba seedlings in trace amounts [35,37].

Furthermore, the production of levopimaradiene was invariably accompanied by the conspicuous appearance of novel peaks eluting later in the chromatogram (Figure 2A, peaks c-e). The extracted mass spectra of these peaks (see Additional file 4) showed characteristic signals at $288 \mathrm{~m} / \mathrm{z}$ (peak c) and $286 \mathrm{~m} / \mathrm{z}$ (peaks d and e). Furthermore, the mass spectrum of peak $\mathrm{c}$ is strongly reminiscent of that of levopimaradiene, and that of peaks $\mathrm{d}$ and e to that of abietatriene. Although the identity of the compounds could not be established, these observations suggests that they correspond to mono-hydroxylated derivatives of levopimaradiene (peak c) and abietatriene (peaks $\mathrm{d}$ and e), respectively. They likely originate from the activity of endogenous hydroxylases from $N$. benthamiana.

\section{Optimization of diterpene production}

A common strategy to increase production of high-value compounds in heterologous expression systems such as E. coli or yeast focused on overexpression of pathway enzymes and improving enzyme activities by codon optimization [12]. Concerning the MEP pathway, it could be shown for example in E. coli that the first step of the pathway, 1-deoxy-D-xylulose-5-phosphate synthase (DXS), constitutes a bottleneck which can be overcome by overexpression [39,40]. A recent example of DXS over-expression in Arabidopsis resulted in a 6-fold increase of taxadiene production compared to the transgenic plant line expressing only taxadiene synthase [41]. Similar effects are reported for geranylgeranyl diphosphate synthase (GGPPS) which uses IPP and DMAPP to synthesize geranylgeranyl diphosphate as the main precursor for a large set of different plastidic isoprenoids. For example, overexpression of GGPPS has been successfully used to boost miltiradiene production in Salvia miltiorrhiza hairy root cultures and in Saccharomyces cerevisiae cultures $[11,42]$. To investigate whether overexpression of the initial step of the MEP pathway or of GGPPS impact CBT-ol production, DXS2 from Solanum lycopersicum [43] and GGPPS2 from Nicotiana tabacum [44] were cloned and expressed alone or in combination with CBTS2a in leaves of $N$. benthamiana. For direct comparison of CBT-ol yield between different assays, the specific agrobacteria mixtures were infiltrated into separate parts on the same leaf. The hexane dipping procedure was used for extraction of CBT-ol from treated tissue. As controls, in planta expression of the individual enzymes, DXS2, p19 and GGPPS2, was performed and as expected did not induce any production of CBT-ol (see Additional file 5).

GC-MS analysis of extracts revealed that co-expression of CBTS2a with DXS2 or GGPPS2 alone does not alter CBT-ol production significantly compared to leaves solely infiltrated with CBTS2a (Figure 3A). In contrast, coexpression of DXS2 and GGPPS2 with CBTS2a resulted in a significant 3.5 -fold increase in the production of CBT-ol (Figure 3A). Under these conditions, the CBT-ol peak was the highest from the whole chromatogram, exceeding the levels of the cuticular lipids which are typically dominant in such extracts (Figure 3B).

\section{Discussion}

Our results demonstrate that the $N$. benthamiana transient expression system described here is a rapid and easily applicable method for testing the functionality of various plant diterpene synthases. NsCBTS2a, RcCS and GbLS were successfully expressed in leaves of $N$. benthamiana, as documented by GC-MS analysis of plant extracts showing the expected product specificity of the recombinant enzymes. Thus, class I as well as bifunctional plant diterpene synthases seem to work equally well in this system. It is intriguing that only GbLS gives rise to significant oxidation products, but it is unlikely that this has something to do with its bifunctionality (see below). Further, it was possible to extract a majority of the reaction products from the surface of infiltrated leaves by organic solvent dipping. The exuded products may derive from agrotreated Nicotiana benthamiana trichomes and epidermal cells whose surface is extracted upon short exposure to hexane. Two types of trichomes were reported in $N$. benthamiana: long stalked glandular hairs with swollen bases and smaller capitate trichomes with thin stalks and single or multiple-celled secretory heads and were demonstrated to be active in acyl sugar synthesis [45]. So far, it is not known whether Agrobacterium would be able to infect trichomes or whether $N$. benthamiana would secrete diterpenoid products into the hairs. However, glandular trichomes from $N$. benthamiana are not known to produce terpenoids. Furthermore, the large amounts of CBT-ol that were observed in the combined expression assays 

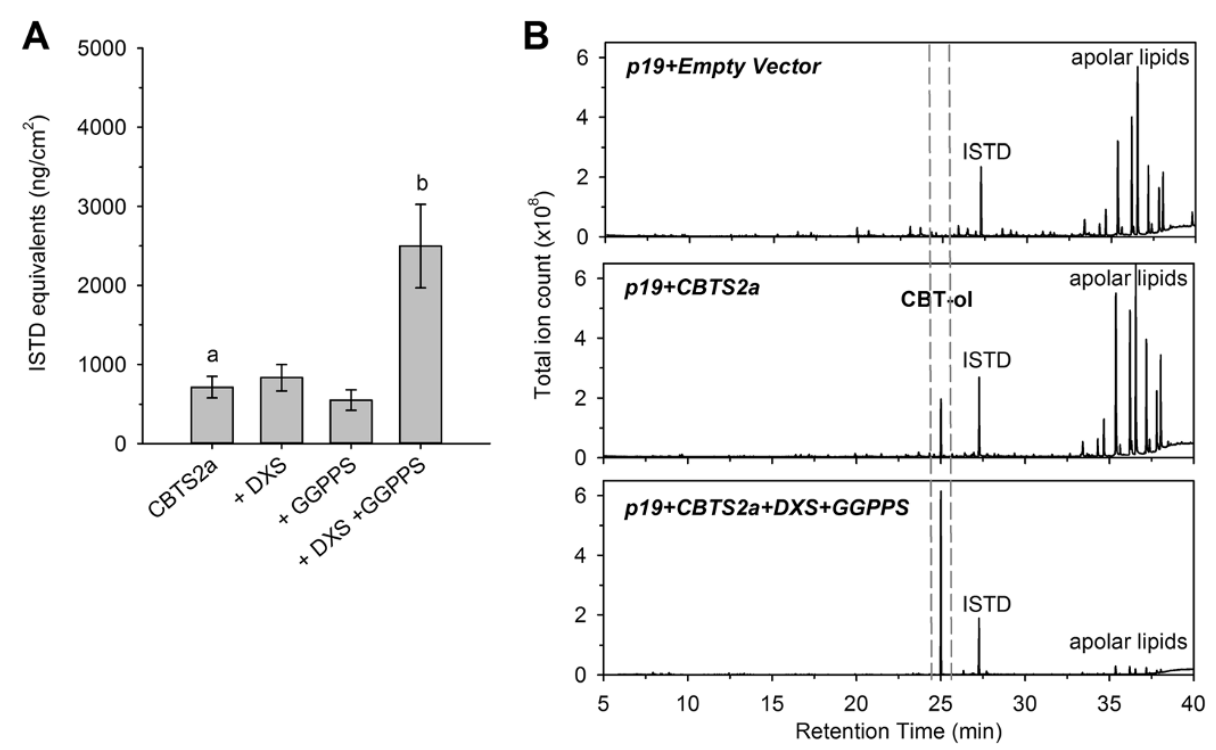

Figure 3 Increasing expression levels of isoprenoid precursor pathway genes DXS and GGPPS lead to increased CBT-ol production in agro-infiltrated $\boldsymbol{N}$. benthamiana leaves. (A) CBT-ol content in hexane washes from N. benthamiana leaves co-expressing p19 and NsCBTS2a with SIDXS2 and/or NtGGPPS2 in different combinations. The appropriate Agrobacterium cultures were infiltrated into separate parts of the same $N$. benthamiana leaf and in two leaves per individual plants. Leaves were harvested five days post-infiltration. Mean CBT-ol values \pm SEM are presented $(n=8)$. Groups indicated by different letters differ significantly from each other regarding their CBT-ol values $(P<0.05$, Student's $t$-test and one-way ANOVA). ISTD - internal standard sclareol; (B) Full total ion chromatograms demonstrating high CBT-ol peak (dashed lines) when NsCBTS2a was co-expressed with both SIDXS2 and NtGGPPS2. No CBT-ol was detected in leaves agro-infiltrated with the empty T-DNA vector as a control.

exceed that of individual cuticular components. These observations suggest that epidermal cells are the main contributors to the diterpenes that are extracted in the leaf washes. In any case, the extraction of leaf surface allows a fast and simple way to identify the products of the expressed diterpene synthases, and could replace recently used labor-extensive methods which include grinding of plant tissue followed by sequential extraction and purification steps prior to chromatography [46]. In addition, because surface extracts are much less complex than whole leaf extracts, this procedure should allow the facile purification of novel products for structure elucidation or for their use as authentic standards for quantification. It should be noted however that this method is likely not to be suitable for smaller terpenes (i.e. mono- and sesquiterpenes) which, because of their volatility, are unlikely to accumulate at the surface of $N$. benthamiana leaves. These can be detected by headspace measurements as was previously shown [30]. With the optimized yields that we obtained for CBT-ol, infiltration of as little as $30 \mathrm{~cm}^{2}$ (one leaf) of $N$. benthamiana would suffice to recover $100 \mu \mathrm{g}$ of CBT-ol, a quantity which is sufficient to produce NMR spectra. Optimization of CBT-ol production was achieved by co-expressing CBTS with both the DXS2 and GGPPS2 enzymes. When synthesis was coupled to the expression of either DXS2 or GGPPS2 alone, the CBT-ol yield did not increase suggesting that the endogenous GGPPS activity is not limiting but would become so when DXS alone is over-expressed. Thus a significant increase in flux through the pathway could only be achieved when both enzymes are over-expressed. It is also possible that the increase of DXS activity could result in the accumulation of intermediates that contribute to negative feedback regulation of the pathway. Methylerythritol cyclodiphosphate (MEcPP), a MEP pathway intermediate, could be such a regulatory compound, since it was recently shown in Arabidopsis to act as a retrograde signal molecule which elicits a stress response [47]. Further analysis of the impact of MEcPP on the expression of MEP pathway genes would need to be carried out to validate this hypothesis. In addition, extensive metabolite profiling of DXP pathway intermediates together with flux analyses would be required to clarify this issue. Moreover, further optimization may be possible by over-expression of other MEP pathway genes, along with DXS and GGPPS.

Although no significant accumulation of by-products could be detected for CBT-ol and casbene, expression of GbLS was accompanied by the modification of levopimaradiene and abietatriene, most likely hydroxylation by endogenous $N$. benthamiana oxidases. To the best of our knowledge, direct modification of exogenous terpene olefins in $N$. benthamiana has not been reported yet. However, hydroxylated or carboxylated terpenoids such as artemisinic acid or germacrenoic acid were shown to 
undergo several types of modifications, including glycosylation and glutathione-transfer [27,30]. The fact that no modification could be detected with casbene or CBT-ol suggests that the modifications are specific to the products of GbLS. One possibility is that one of these products induces a detoxification response in $N$. benthamiana resulting in the expression of oxidases, e. g. P450 mono-oxygenases, which would then hydroxylate levopimaradiene and abietatriene. Alternatively, such oxidases could be constitutively expressed and would exhibit some degree of product specificity since casbene and CBT-ol do not seem to be modified by these activities. Interestingly, such abietane-type diterpenes, including levopimaradiene, are extensively oxidized in various plant species, particularly in the conifers and Lamiaceae, and it is therefore likely that similar processes take place in tobacco [16,48-50]. To further clarify this point, systematic testing of diverse diterpene synthases should indicate to what extent this response is specific of certain diterpenes.

\section{Conclusion}

The characterization of plant diterpene synthases by transient expression in $N$. benthamiana with our optimized method offers a number of advantages over expression in microbial hosts (E. coli or yeast). First, subcellular targeting is not an issue any more since the processing of organellar targeting signals is highly conserved in plants. Thus, full length coding sequences can be used directly for expression without concerns about removing a signal peptide whose length still has to be determined empirically. Second, solubility and functionality of the expressed protein is often an issue when expressing plant enzymes in $E$. coli or yeast, particularly with terpene synthases. Here, although the diterpene synthases tested come from highly divergent plant species, they could all be functionally expressed without optimization of the coding sequence. Finally, in comparison to transgenic plants, the transient system provides a rapid method, reduced costs of production and scalability thanks to the large amounts of tissue that can be infiltrated. Co-expression with SIDXS2 and NtGGPPS2 ensures that sufficient quantity of the product for structure elucidation can be recovered with a relatively small sample size, i.e. one leaf. Beyond the characterization of diterpene synthase products, our optimized method should be of general use for the reconstitution of diterpenoid biosynthetic pathways and combinatorial biosynthesis.

\section{Methods}

Plant material

Nicotiana benthamiana seeds were germinated directly in soil and grown in climate-controlled chambers with a 16-h day/8-h night photoperiod under an illumination of
$90 \mu \mathrm{mol} / \mathrm{s} / \mathrm{m}^{2}$ generated by MT250DL metal halide lamps. Temperatures in the growth chamber were set to $20^{\circ} \mathrm{C}$ during the day and to $18^{\circ} \mathrm{C}$ in the night. Humidity was adjusted to $60 \%$.

\section{Oligonucleotide primers}

All oligonucleotide primers which were used for the isolation of cDNA for cloning and in PCR reactions are listed in Additional file 6 . The cloning primers were designed by hand with artificial BpiI restriction sites at the 5' and 3' end necessary for Golden Gate cloning [51] procedures.

\section{T-DNA constructs for in planta expression}

Recombinant plasmids were generated according to the Golden Gate cloning method [51,52]. Full length coding sequences for NsCBTS2a, RcCS, GbLS, SIDXS2 and NtGGPPS2 were PCR amplified using Phusion Hot Start II High-Fidelity DNA Polymerase (Thermo Scientific, http://www.thermoscientific.com) and gene specific primers. Prior to cloning, any BpiI and BsaI restriction sites that were present within gene sequence were removed by mutating single nucleotides of enzyme recognition pattern and dividing the gene into sub-fragments devoid of these sites according to [51]. Entry modules were generated by cloning the sub-fragments into vector pGEM-T-easy (Promega, http://www.promega.com) according to the manufacturer's instructions or in pUC18 according to Engler and Marillonnet [53]. The entry modules were assembled to full length gene into Golden Gate entry vector pL0-SC [54] using BpiI (Fermentas, http://www. thermoscientificbio.com/fermentas) and Promega T4-HCLigase, and the inserted fragment was verified by sequencing. Finally, the target genes in pL0-SC were transferred into T-DNA vector pL1F-1, and fused to CaMV $35 \mathrm{~S}$ promoter and Nos-terminator by a single reaction using BsaI (New England Biolabs, http://www.neb.com) and T4-HC-Ligase (Promega).

\section{Transient expression in Nicotiana benthamiana}

T-DNA plasmids containing diTPS gene insertion were transformed into Agrobacterium tumefaciens strain GV3101::pMP90 [54] by electroporation. The agrobacteria were streaked on LB agar plates containing rifampicin $(25 \mu \mathrm{g} / \mathrm{ml})$, gentamycin $(50 \mu \mathrm{g} / \mathrm{ml})$ and carbenicillin $(50 \mu \mathrm{g} / \mathrm{ml})$. The plates were incubated for 2-3 days at $28^{\circ} \mathrm{C}$ and colonies were tested for insertion by PCR using DreamTaq DNA Polymerase (Thermo Scientific) and gene specific primers. Transformed single colony was used to inoculate a $5 \mathrm{ml}$ culture of LB medium plus appropriate antibiotics and cells were grown for 24 hours at $28^{\circ} \mathrm{C}$ and $180 \mathrm{rpm}$. This pre-culture was used to inoculate a $50 \mathrm{ml}$ culture and grown for another $16-20$ hours at $28^{\circ} \mathrm{C}$. The OD $(600 \mathrm{~nm})$ was 
adjusted to 0.5 in a total volume of $20 \mathrm{ml}$. Agrobacteria were harvested by centrifugation at $1800 \times g$ and $4^{\circ} \mathrm{C}$ for $90 \mathrm{~min}$. When several constructs were coinfiltrated, the corresponding agrobacteria batches were mixed together in equal proportion before centrifugation and so that the $\mathrm{OD}_{600}=0.5$. The cell pellet was resuspended in 0.25 volumes LB medium, 0.25 volumes sterile water and 0.5 volumes of infiltration buffer $(10 \% \mathrm{w} / \mathrm{v}$ sucrose, $20 \mathrm{mM}$ glucose, $8.6 \mathrm{~g} / \mathrm{l}$ Murashige \& Skoog basal salt mixture) to reach a final volume of $20 \mathrm{ml} .20 \mu \mathrm{M}$ Acetosyringone were added to the agrobacteria suspension and the mixture was immediately infiltrated into leaves of 4-5 weeks old $N$. benthamiana plants by pressing a $1 \mathrm{ml}$ syringe against the abaxial side of the leaf. Transformed plants were returned to climate-controlled chambers for 3-5 days until further analysis. Agrobacterium strain GV3101 carrying a T-DNA expressing the p19 protein was co-infiltrated to improve transgene expression by suppressing post-transcriptional gene silencing [26].

\section{Metabolite extraction}

To isolate diterpenoid compounds produced in leaves agro-infiltrated with diTPS expression constructs, six leaf discs $(9 \mathrm{~mm}$ in diameter) were prepared from infiltrated leaf area and extracted by dipping in $1 \mathrm{ml}$ hexane for $2 \mathrm{~min}$ at room temperature. The extract was evaporated completely under a stream of nitrogen, and resuspended in $200 \mu \mathrm{l}$ of hexane supplied with $20 \mu \mathrm{M}$ of internal standard sclareol (Sigma-Aldrich, http:// www.sigmaaldrich.com). The extracts were centrifuged at $16.000 \times g$ for $5 \mathrm{~min}$ prior to their transfer into GC vials.

After having extracted the leaf surface, the leaf discs were transferred into test tubes (Biozym Scientific, http:// www.biozym.com) containing five steel beads $(3 \mathrm{~mm}$ in diameter), and quickly frozen in liquid nitrogen. Frozen leaf discs were homogenized to a fine powder using Mixer Mill MM-400 (Retsch, http://www.retsch.de) and two runs at $30 \mathrm{~Hz}$ for $30 \mathrm{sec}$, and followed by using FastPrep-24 instrument (MP Biomedicals, http://www.mpbio.com) for $20 \mathrm{sec}$ at $4 \mathrm{~m} / \mathrm{s}$ with the samples stored on dry ice. Samples were mixed with $1 \mathrm{ml}$ hexane and extraction was carried out via FastPrep-24 system for $20 \mathrm{sec}$ at $6 \mathrm{~m} / \mathrm{s}$, once with the samples stored on dry ice and two more times at room temperature. The extracts were clarified by centrifugation at $16.000 \times g$ and $4^{\circ} \mathrm{C}$ for $15 \mathrm{~min}$, and further purified using unmodified Chromabond-SiOH SPE columns (Macherey-Nagel, http://www.mn-net.com). The SPE column was equilibrated with one volume of hexane, followed by the loading of the sample extract. The column was washed with $1 \mathrm{ml}$ hexane. Afterwards, five fractions of each $1 \mathrm{ml}$ were collected using hexane/ethyl-acetate/ methanol (90:10:1 by volume) as eluent. The fractions containing the product of interest (see Additional file 2) were combined and the eluate was concentrated under nitrogen flow in a $1.5 \mathrm{~mL}$ microcentrifuge tube, and the residue was dissolved with $400 \mu \mathrm{l}$ hexane. Before transferring the extracts into $\mathrm{GC}$ vials, the samples were centrifuged at $16.000 \times g$ for $2 \mathrm{~min}$ and mixed with internal standard sclareol giving a final concentration of $20 \mu \mathrm{M}$.

\section{GC-MS analysis of solvent extracts}

For GC-MS analysis, $1 \mu \mathrm{L}$ of the samples was injected directly into a Trace GC Ultra gas chromatograph coupled to ISQ mass spectrometer (Thermo Scientific). Separation was achieved in a $30-\mathrm{m} \times 0.32-\mathrm{mm}$ diameter capillary, with a $0.25-\mu \mathrm{m}$ film of ZB-5 ms (Phenomenex, http:// www.phenomenex.com). Splitless mode was used for the injector with the inlet temperature set to $250^{\circ} \mathrm{C}$. The oven was programmed to start at $50^{\circ} \mathrm{C}$ and a 1 -min hold, after which temperature increased to $300^{\circ} \mathrm{C}$ at a rate of $7^{\circ} \mathrm{C}$ $\min ^{-1}$ and further to $330^{\circ} \mathrm{C}$ with $20^{\circ} \mathrm{C} \mathrm{min}^{-1}$ and a 5 -min hold. Helium was used as carrier gas and was adjusted to a flow rate of $1 \mathrm{ml} / \mathrm{min}$. Electron impact was recorded at $70 \mathrm{eV}$ and MS data were collected from 50 to $450 \mathrm{~m} / \mathrm{z}$ during the temperature ramp. Diterpenoids were quantified by determination of their total ion count (TIC) peak area and comparison to the peak area of the internal standard (ISTD) sclareol. The amount of target compounds is given as ISTD equivalents.

\section{Accession numbers}

Sequence data from this article can be found in public databases under the following accession numbers: [GenBank:HM241151 (NsCBTS2a), GenBank:L32134 (RcCS), GenBank:AF331704 (GbLS), GenBank:FN424052 (SIDXS2), GenBank:GQ911584 (NtGGPPS2)].

\section{Additional files}

Additional file 1: Mass spectra recorded for the main products of the diterpene synthases investigated in this study: (A), CBT-ol, measured mass spectrum and published reference mass spectra; (B), Levopimaradiene; and (C) Casbene.

Additional file 2: GC-MS analysis of SPE eluates from total extracts of $N$. benthamiana leaves agro-infiltrated with $p 19$ and $N s C B T S 2 a$. Treated leaves were harvested five days post-infiltration and crude extracts from six frozen leaf discs ( $9 \mathrm{~mm}$ in diameter) were purified over SPE column using hexane/ethyl-acetate/methanol (90:10:1, v/V) as eluent. The CBT-ol peak is framed by vertical dashed lines.

Additional file 3: GC-MS analysis of total extracts and hexane washes of $N$. benthamiana leaves co-expressing $p 19$ and the empty T-DNA vector (pL1F-1) as a control. Treated leaves were harvested five days after infiltration of the plants. A) $272 \mathrm{~m} / \mathrm{z}$ extracted spectra of SPE eluates from total leaf extracts after purification over SPE column. B) $272 \mathrm{~m} / \mathrm{z}$ extracted total ion chromatograms of hexane washes from three independent leaf samples. No specific peak for $272 \mathrm{~m} / \mathrm{z}$ was detected in the extracts indicating that no CBT-ol, casbene or levopimaradiene can be found in $N$. benthamiana upon co-expression of p19 and the empty T-DNA vector.

Additional file 4: GC-MS analysis of reaction products a - e which occurred in N. benthamiana leaves agro-infiltrated with $p 19$ and 
GbLS. (A) $272 \mathrm{~m} / \mathrm{z}$ extracted ion chromatograms and full scan mass spectra for diterpene-like compound peaking at $23.97 \mathrm{~min}$ (peak a). (B) $270 \mathrm{~m} / \mathrm{z}$ extraction for peak b which was identified as Abietatriene using NIST MS search software v2.0 (http://chemdata.nist.gov/) (C) $288 \mathrm{~m} / \mathrm{z}$ extracted chromatogram and corresponding mass spectrum for product peak c eluting after $27.88 \mathrm{~min}$. (D) $286 \mathrm{~m} / \mathrm{z}$ extracted ion chromatograms and the mass spectra recorded for compounds detected at $28.33 \mathrm{~min}$ (peak d) and $28.49 \mathrm{~min}$ (peak e), respectively.

Additional file 5: Sections of total ion chromatograms obtained by GC-MS analysis of hexane washes from leaves infiltrated with $p 19$ alone or co-infiltrated with $p 19$ and SIDXS2 or NtGGPPS2 as controls. The infiltrated leaves were harvested five days post-infiltration.

Additional file 6: List of oligonucleotide primers which were used for PCR and Golden Gate cloning procedures.

\section{Competing interests}

The authors declare that they have no competing interests.

\section{Authors' contributions}

AT conceived the study, KB and AT designed the experiments, KB carried out the experiments, $\mathrm{KB}$ and $\mathrm{AT}$ interpreted the results and wrote the manuscript. KB and AT read and approved the final manuscript. AT submitted the manuscript.

\section{Acknowledgments}

K.B. was funded by E.U. grant agreement KBBE-227448 for the TERPMED project (http://www.terpmed.eu)

Received: 8 October 2013 Accepted: 2 December 2013

Published: 12 December 2013

\section{References}

1. Guerra-Bubb J, Croteau R, Williams RM: The early stages of taxol biosynthesis: an interim report on the synthesis and identification of early pathway metabolites. Nat Prod Rep 2012, 29:683-696.

2. Bohlmann J, Keeling Cl: Terpenoid biomaterials. Plant J 2008, 54:656-669.

3. Tounekti T, Munne-Bosch S: Enhanced phenolic diterpenes antioxidant levels through non-transgenic approaches. Critic Rev Plant Sci 2012, 31:505-519

4. Degenhardt J, Kollner TG, Gershenzon J: Monoterpene and sesquiterpene synthases and the origin of terpene skeletal diversity in plants. Phytochemistry 2009, 70:1621-1637.

5. Koeksal M, Jin Y, Coates RM, Croteau R, Christianson DW: Taxadiene synthase structure and evolution of modular architecture in terpene biosynthesis. Nature 2011, 469:116-120

6. Huang EX, Huang QL, Wildung MR, Croteau R, Scott Al: Overproduction, in Escherichia coli, of soluble taxadiene synthase, a key enzyme in the taxol biosynthetic pathway. Prot Express Purif 1998, 13:90-96.

7. Hall DE, Zerbe P, Jancsik S, Quesada AL, Dullat H, Madilao LL, Yuen M, Bohlmann J: Evolution of conifer diterpene synthases: diterpene resin acid biosynthesis in lodgepole pine and jack pine involves monofunctional and bifunctional diterpene synthases. Plant Physiol 2013, 161:600-616.

8. Sallaud C, Giacalone C, Toepfer R, Goepfert S, Bakaher N, Roesti S, Tissier A: Characterization of two genes for the biosynthesis of the labdane diterpene Z-abienol in tobacco (Nicotiana tabacum) glandular trichomes. Plant J 2012, 72:1-17.

9. Hill AM, Cane DE, Mau CJD, West CA: High level expression of Ricinus communis casbene synthase in Escherichia coli and characterization of the recombinant enzyme. Arch Biochem Biophys 1996, 336:283-289.

10. Reiling KK, Yoshikuni Y, Martin VJJ, Newman J, Bohlmann J, Keasling JD: Mono and diterpene production in Escherichia coli. Biotechnol Bioeng 2004, 87:200-212.

11. Zhou YJ, Gao W, Rong Q, Jin G, Chu H, Liu W, Yang W, Zhu Z, Li G, Zhu G, et al: Modular pathway engineering of diterpenoid synthases and the mevalonic acid pathway for miltiradiene production. J Am Chem Soc 2012, 134:3234-3241.
12. Muntendam R, Melillo E, Ryden A, Kayser O: Perspectives and limits of engineering the isoprenoid metabolism in heterologous hosts. Appl Microbiol Biotechnol 2009, 84:1003-1019.

13. Pitera DJ, Paddon CJ, Newman JD, Keasling JD: Balancing a heterologous mevalonate pathway for improved isoprenoid production in Escherichia coli. Metab Eng 2007, 9:193-207.

14. Anthony JR, Anthony LC, Nowroozi F, Kwon G, Newman JD, Keasling JD: Optimization of the mevalonate-based isoprenoid biosynthetic pathway in Escherichia coli for production of the anti-malarial drug precursor amorpha-4,11-diene. Metab Eng 2009, 11:13-19.

15. Tsuruta H, Paddon CJ, Eng D, Lenihan JR, Horning T, Anthony LC, Regentin R, Keasling JD, Renninger NS, Newman JD: High-level production of amorpha-4,11-diene, a precursor of the antimalarial agent artemisinin, in Escherichia coli. PLOS ONE 2009, 4:e4489.

16. Guo J, Zhou YJ, Hillwig ML, Shen Y, Yang L, Wang Y, Zhang X, Liu W, Peters RJ, Chen $X$, et al: CYP76AH1 catalyzes turnover of miltiradiene in tanshinones biosynthesis and enables heterologous production of ferruginol in yeasts. Proc Natl Acad Sci U S A 2013, 110:12108-12113.

17. Dai Z, Liu Y, Huang L, Zhang X: Production of miltiradiene by metabolically engineered Saccharomyces cerevisiae. Biotechnol Bioeng 2012, 109:2845-2853.

18. Caniard A, Zerbe P, Legrand S, Cohade A, Valot N, Magnard JL, Bohlmann J, Legendre L: Discovery and functional characterization of two diterpene synthases for sclareol biosynthesis in Salvia sclarea (L.) and their relevance for perfume manufacture. BMC Plant Biol 2012, 12:119.

19. Hamberger B, Ohnishi T, Seguin A, Bohlmann J: Evolution of diterpene metabolism: Sitka spruce CYP720B4 catalyzes multiple oxidations in resin acid biosynthesis of conifer defense against insects. Plant Physiol 2011, 157:1677-1695.

20. Kirby J, Nishimoto M, Park JG, Withers ST, Nowroozi F, Behrendt D, Rutledge EJ, Fortman JL, Johnson HE, Anderson JV, Keasling JD: Cloning of casbene and neocembrene synthases from Euphorbiaceae plants and expression in Saccharomyces cerevisiae. Phytochemistry 2010, 71:1466-1473.

21. Engels B, Dahm P, Jennewein S: Metabolic engineering of taxadiene biosynthesis in yeast as a first step towards Taxol (Paclitaxel) production. Metab Eng 2008, 10:201-206.

22. Pateraki I, Kanellis AK: Isolation and functional analysis of two Cistus creticus CDNAs encoding geranylgeranyl diphosphate synthase. Phytochemistry 2008, 69:1641-1652.

23. Paddon CJ, Westfall PJ, Pitera DJ, Benjamin K, Fisher K, McPhee D, Leavel MD, Tai A, Main A, Eng D, et al: High-level semi-synthetic production of the potent antimalarial artemisinin. Nature 2013, 496:528-532.

24. Goodin MM, Zaitlin D, Naidu RA, Lommel SA: Nicotiana benthamiana: its history and future as a model for plant-pathogen interactions. Mol Plant-Microbe Interact 2008, 21:1015-1026.

25. Kapila J, DeRycke R, VanMontagu M, Angenon G: An Agrobacterium-mediated transient gene expression system for intact leaves. Plant Sci 1997, 124:227.

26. Voinnet O, Rivas S, Mestre P, Baulcombe D: An enhanced transient expression system in plants based on suppression of gene silencing by the p19 protein of tomato bushy stunt virus. Plant J 2003, 33:949-956.

27. van Herpen TWJM, Cankar K, Nogueira M, Bosch D, Bouwmeester HJ, Beekwilder J: Nicotiana benthamiana as a production platform for artemisinin precursors. PLOS ONE 2010, 5:e14222.

28. Kanagarajan S, Muthusamy S, Gliszczynska A, Lundgren A, Brodelius PE: Functional expression and characterization of sesquiterpene synthases from Artemisia annua L. using transient expression system in Nicotiana benthamiana. Plant Cell Rep 2012, 31:1309-1319.

29. Green $S A$, Chen XY, Matich AJ: In planta transient expression analysis of monoterpene synthases. In Natural Product Biosynthesis by Microorganisms and Plants, Part A. Methods in Enzymology. Volume 515. Edited by Hopwood DA. San Diego: Elsevier Academic Press; 2012:43-61.

30. Liu Q, Majdi M, Cankar K, Goedbloed M, Charnikhova T, Verstappen FWA, de Vos RCH, Beekwilder J, van der Krol S, Bouwmeester HJ: Reconstitution of the costunolide biosynthetic pathway in yeast and nicotiana benthamiana. PLOS ONE 2011, 6:e23255.

31. Mugford ST, Qi X, Bakht S, Hill L, Wegel E, Hughes RK, Papadopoulou K, Melton R, Philo M, Sainsbury F, et al: A serine carboxypeptidase-like acyltransferase is required for synthesis of antimicrobial compounds and disease resistance in oats. Plant Cell 2009, 21:2473-2484.

32. Geisler K, Hughes RK, Sainsbury F, Lomonossoff GP, Rejzek M, Fairhurst S, Olsen C-E, Motawia MS, Melton RE, Hemmings AM, et al: Biochemical 
analysis of a multifunctional cytochrome P450 (CYP51) enzyme required for synthesis of antimicrobial triterpenes in plants. Proc Natl Acad Sci U S A 2013, 110:E3360-E3367.

33. Ennajdaoui H, Vachon G, Giacalone C, Besse I, Sallaud C, Herzog M, Tissier A: Trichome specific expression of the tobacco (Nicotiana sylvestris) cembratrien-ol synthase genes is controlled by both activating and repressing cis-regions. Plant Mol Biol 2010, 73:673-685.

34. Mau CJD, West CA: Cloning of casbene synthase CDNA - evidence for conserved structural features among terpenoid cyclases in plants. Proc Natl Acad Sci U S A 1994, 91:8497-8501.

35. Schepmann HG, Pang JH, Matsuda SPT: Cloning and characterization of Ginkgo biloba levopimaradiene synthase, which catalyzes the first committed step in ginkgolide biosynthesis. Arch Biochem Biophys 2001, 392:263-269.

36. Ro DK, Bohlmann J: Diterpene resin acid biosynthesis in loblolly pine (Pinus taeda): functional characterization of abietadiene/levopimaradiene synthase (PtTPS-LAS) CDNA and subcellular targeting of PtTPS-LAS and abietadienol/abietadienal oxidase (PtAO, CYP720B1). Phytochemistry 2006, 67:1572-1578

37. Neau E, Cartayrade A, Balz JP, Carde JP, Walter J: Ginkgolide and bilobalide biosynthesis in Ginkgo biloba.2. Identification of a possible intermediate compound by using inhibitors of cytochrome P-450-dependent oxygenases. Plant Physiol Biochem 1997, 35:869-879.

38. Leonard E, Ajikumar PK, Thayer K, Xiao W-H, Mo JD, Tidor B, Stephanopoulos G, Prather KL: Combining metabolic and protein engineering of a terpenoid biosynthetic pathway for overproduction and selectivity control. Proc Nat Acad Sci U S A 2010, 107:13654-13659.

39. Wang HH, Isaacs FJ, Carr PA, Sun ZZ, Xu G, Forest CR, Church GM: Programming cells by multiplex genome engineering and accelerated evolution. Nature 2009, 460:894-898.

40. Ajikumar PK, Xiao W-H, Tyo KEJ, Wang Y, Simeon F, Leonard E, Mucha O, Phon TH, Pfeifer B, Stephanopoulos G: Isoprenoid pathway optimization for taxol precursor overproduction in Escherichia coli. Science 2010, 330:70-74.

41. Botella-Pavia P, Besumbes O, Phillips MA, Carretero-Paulet L, Boronat A, Rodriguez-Concepcion M: Regulation of carotenoid biosynthesis in plants: evidence for a key role of hydroxymethylbutenyl diphosphate reductase in controlling the supply of plastidial isoprenoid precursors. Plant J 2004, 40:188-199.

42. Kai G, Xu H, Zhou C, Liao P, Xiao J, Luo X, You L, Zhang L: Metabolic engineering tanshinone biosynthetic pathway in Salvia miltiorrhiza hairy root cultures. Metab Eng 2011, 13:319-327.

43. Paetzold H, Garms S, Bartram S, Wieczorek J, Uros-Gracia E-M, RodriguezConcepcion M, Boland W, Strack D, Hause B, Walter MH: The isogene 1-deoxy-d-xylulose 5-phosphate synthase 2 controls isoprenoid profiles, precursor pathway allocation, and density of tomato trichomes. Mol Plant 2010, 3:904-916.

44. Orlova I, Nagegowda DA, Kish CM, Gutensohn M, Maeda H, Varbanova M, Fridman E, Yamaguchi S, Hanada A, Kamiya Y, et al: The small subunit of snapdragon geranyl diphosphate synthase modifies the chain length specificity of tobacco geranylgeranyl diphosphate synthase in planta. Plant Cell 2009, 21:4002-4017.

45. Slocombe SP, Schauvinhold I, McQuinn RP, Besser K, Welsby NA, Harper A, Aziz N, Li Y, Larson TR, Giovannoni J, et al: Transcriptomic and reverse genetic analyses of branched-chain fatty acid and acyl sugar production in solanum pennellii and nicotiana benthamiana. Plant Physiol 2008, 148:1830-1846.

46. Zerbe $\mathrm{P}$, Hamberger B, Yuen MM, Chiang A, Sandhu HK, Madilao LL, Nguyen A, Bach SS, Bohlmann J: Gene discovery of modular diterpene metabolism in nonmodel systems. Plant Physiol 2013, 162:1073-1091.

47. Xiao Y, Savchenko T, Baidoo EE, Chehab WE, Hayden DM, Tolstikov V, Corwin JA, Kliebenstein DJ, Keasling JD, Dehesh K: Retrograde signaling by the plastidial metabolite MECPP regulates expression of nuclear stress-response genes. Cell 2012, 149:1525-1535.

48. Funk $C$, Croteau R: Diterpenoid resin acid biosynthesis in conifers: characterization of two cytochrome P450-dependent monooxygenases and an aldehyde dehydrogenase involved in abietic acid biosynthesis. Arch Biochem Biophys 1994, 308:258-266.

49. Ro DK, Arimura G, Lau SY, Piers E, Bohlmann J: Loblolly pine abietadienol/ abietadienal oxidase PtAO (CYP720B1) is a multifunctional, multisubstrate cytochrome P450 monooxygenase. Proc Natl Acad Sci U S A 2005, 102:8060-8065.

50. Zi J, Peters RJ: Characterization of CYP76AH4 clarifies phenolic diterpenoid biosynthesis in the Lamiaceae. Org Biomol Chem 2013, 11:7650-7652.

51. Engler C, Kandzia R, Marillonnet S: A one pot, one step, precision cloning method with high throughput capability. PLOS ONE 2008, 3:e3647.

52. Weber E, Engler C, Gruetzner R, Werner S, Marillonnet S: A modular cloning system for standardized assembly of multigene constructs. PLOS ONE 2011, 6:e16765.

53. Engler C, Marillonnet $\mathrm{S}$ : Generation of families of construct variants using golden gate shuffling. Methods Mol Biol 2011, 729:167-181.

54. Koncz C, Schell J: The promoter of TL-DNA gene 5 controls the tissuespecific expression of chimeric genes carried by a novel type of Agrobacterium binary vector. Mol Gen Genet 1986, 204:383-396.

doi:10.1186/1746-4811-9-46

Cite this article as: Brückner and Tissier: High-level diterpene production by transient expression in Nicotiana benthamiana. Plant Methods 2013 9:46.

\section{Submit your next manuscript to BioMed Central and take full advantage of:}

- Convenient online submission

- Thorough peer review

- No space constraints or color figure charges

- Immediate publication on acceptance

- Inclusion in PubMed, CAS, Scopus and Google Scholar

- Research which is freely available for redistribution 